\title{
Stress- Rest Myocardial Perfusion Scintigraphy and Adverse Cardiac Events in Heart Failure Patients
}

\author{
Amalia Peix MD PhD DrSc, Yanisbel Macides MD, Lydia Rodríguez, Lázaro O. Cabrera MD, Kenia Padrón MD, \\ Flor Heres MD MS, Dania Consuegra, Yoel Fernández, Erick Mena
}

\begin{abstract}
INTRODUCTION

Heart failure, primarily in the elderly, is a growing epidemic in today's world. It leads to high rates of disability and mortality, as well as significant health care expenditures, making it important to assess possible predictors of adverse cardiac events. In Cuba, heart failure mortality is $19.1 / 100,000$ population.
\end{abstract}

OBJECTIVES Assess the value of stress-rest protocol gated-SPECT for identifying patients with symptomatic heart failure likely to suffer adverse cardiac events.

METHODS A study was conducted of 52 patients (mean age 59 years, SD 9; $62 \%$ women) with functional capacity II/III (New York Heart Association scale) and left ventricular ejection fraction $<40 \%$. Patients were divided into two groups based on coronary heart disease diagnosis: those with coronary heart disease (41), labeled ischemic; and those without (11), labeled nonischemic. All underwent gated SPECT myocardial perfusion scintigraphy with technetium-99m-labeled methoxyisobutyl isonitrile, using a two-day stress-rest protocol, including evaluation of intraventricular synchrony by phase analysis. Patients were followed over 36 months for adverse cardiac effects.

RESULTS No significant differences were observed between the two groups during the stress test with regard to exercise time, metabol- ic equivalents or percentage of maximal heart rate during maximal stress. Summed stress, rest and difference scores, however, were significantly different between the ischemic and nonischemic groups: 16.82 (SD 6.37) vs. 7.54 (SD 5.8), $p<0.001 ; 14.43$ (SD 6.28) vs. 6.45 (SD 3.77), $p=0.001$; and 2.39 (SD 4.89) vs. 1.09 (SD 3.7), $p$ $=0.034$. No differences were found in ventricular function, although stress-minus-rest left ventricular ejection fraction was slightly lower in patients with ischemic heart disease $(-1.29$, SD 5.8) than in patients without ischemic heart disease (1.27, SD 4.31). Dyssynchrony was greater in patients with ischemic heart disease than in those without, primarily during stress $(p<0.01)$. The only variable that showed a possible association with the occurrence of adverse events was $<5$ metabolic equivalents on the stress test $(p=0.03)$, while resting phase SD showed only a tendency toward association $(p=0.05)$.

CONCLUSIONS Information on myocardial perfusion, functional capacity and intraventricular synchrony obtained from stress-rest gated SPECT may help identify patients with symptomatic heart failure who are likely to develop adverse cardiac events, enabling better management of higher-risk cases and improved allocation of resources.

KEYWORDS Heart failure, congestive heart failure, myocardial ischemia, ischemic heart disease, coronary artery disease, gated SPECT, perfusion, ventricular function, intraventricular synchrony, phase analysis, Cuba

\section{INTRODUCTION}

Heart failure (HF) is a growing epidemic worldwide, affecting over 15 million people and accounting for about $5 \%$ of all hospital admissions.[1] Globally, ischemic heart disease (IHD) is the main cause of HF $(60 \%-75 \%$ of cases).[2] HF prevalence in the adult population ranges from $1 \%$ to $2 \%$, highest in the elderly and resulting in considerable disability, mortality and high health care expenditures.[3] Thus, proper diagnosis and prognosis assessment of heart failure is vital to patients, health care providers and health systems. This is also true in Cuba, where HF prevalence is $1.6 \%$ in the population aged $>15$ years $(5 \%$ in persons aged $65-74$ years, increasing to $10.7 \%$ at ages $\geq 75$ years).[4]

Myocardial perfusion scintigraphy using gated single-photon emission-computed tomography (gated SPECT) is the only noninvasive imaging technique that provides, in a single study, reproducible overall and regional information on ventricular function, myocardial perfusion and presence or absence of intraventricular synchrony. The prognostic value of perfusion defects found through gated SPECT has been properly validated.[5,6] Phase analysis has been applied in gated SPECT in recent years for assessing intraventricular mechanical synchrony, $[7,8]$ and phase SD and histogram bandwidth have been recognized as the main indicators for measuring intraventricular synchrony.[9]

Several studies have been published on the usefulness of SPECT imaging in assessing HF patients. [5,10-13] Not enough research has been done, however, to enable analysis of the relationship between the extent of perfusion defects found by nuclear techniques and disease course in HF patients with dilated cardiomyopathy, as well as the value for these patients' prognosis of the dyssynchrony indices from phase analysis (phase SD and histogram bandwidth), taking into particular account changes that exercise may produce in these parameters.

Our group recently completed a study of patients with dilated cardiomyopathy and left ventricular ejection fraction (LVEF) $<40 \%$ who underwent gated SPECT at rest and were followed for 39 months. The results showed phase SD, ischemic etiology and summed resting score (SRS) (these attained the highest odds ratios: $1.91,1.66$ and 1.55 , respectively) to be the best predictors for event occurrence (progression of HF, potentially lethal ventricular arrhythmia, death, or acute coronary syndrome). Only 5 patients who had heart events were nonischemic, whereas 39 $(89 \%)$ were ischemic.[14]

The purpose of this study was to assess the value of stress-rest protocol gated SPECT-the information it provides on myocardial perfusion, ventricular function and intraventricular synchronyfor identifying symptomatic HF patients who are likely to develop adverse cardiac events.

\section{METHODS}

Population The study universe comprised 52 patients (mean age 59 years, SD 9; 62\% women) referred by their attending physicians to the Nuclear Medicine Department of the Cardiology 
and Cardiovascular Surgery Institute (ICCCV) in Havana, Cuba, between December 2010 and April 2012. Inclusion criteria were age $\geq 25$ years, with functional capacity II/III (New York Heart Association scale), LVEF $<40 \%$ and capable of exercise. Exclusion criteria were pregnancy, complete left bundle branch block, pacemaker implanted before the nuclear study, and arrhythmia that could prevent gating.

Design Patients were divided into two groups: those already diagnosed with IHD (41 patients, labeled ischemic) and those without IHD (11 patients, labeled nonischemic). Patients were classified as having HF of ischemic etiology based on a history, in the six months prior to inclusion in the study, of coronary angiography with significant epicardial coronary stenosis $(>50 \%$ reduction of vascular lumen); acute coronary syndrome with or without ST elevation (STEMI or NSTEMI, respectively); or percutaneous transluminal coronary angioplasty.

Upon inclusion in the study, patient data on the following coronary risk factors were obtained from the attending physician's clinical history: hypertension, diabetes mellitus, dyslipidemia, smoking (current or past) and obesity.

Myocardial perfusion scintigraphy Gated SPECT was performed in all cases, using a two-day stress-rest protocol. The symptom-limited treadmill stress test (MTM-1500 med, Schiller, Switzerland) was performed the first day. Stress-test variables were presence or absence of angina with stress, ST depression $\geq 2 \mathrm{~mm}$ with stress on electrocardiogram, occurrence of cardiac arrhythmia during stress, percentage of maximal heart rate attained (220 - age), duration of exercise in minutes, and number of metabolic equivalents (MET) attained on maximal exertion. At maximal exertion, a $20 \mathrm{mCi}$ dose of technetium$99 \mathrm{~m}$-labeled methoxyisobutyl isonitrile ( $\left.{ }^{99 \mathrm{~m}} \mathrm{Tc}-\mathrm{MIBI}\right)$ was injected and the patient continued exercising for an additional 60-90 seconds.

Post-stress images were taken 45-60 minutes following tracer injection, with the patient remaining supine. A dual-head gamma camera (Nucline Spirit DHV, Mediso, Hungary) was used, equipped with low-energy collimators, high-resolution and parallel holes, with an energy emission window of $20 \%$, centered on the $140 \mathrm{keV}$ energy peak. A total of 64 projections were obtained (20 seconds per projection), 8 frames/cycle, with a 64 × 64 matrix, in a $180^{\circ}$ orbit. The following day, resting images were obtained one hour following injection of $20 \mathrm{mCi}{ }^{99 m} \mathrm{Tc}-\mathrm{MIBI}$. Tomographic images were reconstructed using filtered back-projection with a Butterworth filter, order 7 , and a frequency of 0.25 cycles/pixel. Neither attenuation nor dispersion was corrected.

Interpretation of scintigraphic images For visual semiquantitative interpretation, short- and long-axis vertical tomograms were used, divided into 17 segments.[15] Each segment was assessed by consensus of two independent expert observers uninformed of the clinical and echocardiographic data, using a 5-point scale: $0=$ normal, 1 = slightly reduced uptake (slight defect), 2 = moderately reduced uptake (moderate defect), $3=$ severely reduced uptake, 4 = absence of myocardial uptake of the radiopharmaceutical. Scores of 3 and 4 correspond to severe defects. Segments with uptake reduction (perfusion defects on stress) were considered reversible perfusion defects if the score dropped $\geq 1$ point from stress to resting.
Perfusion variables were summed stress score (SSS) and summed rest score (SRS), calculated by the sum of the scores for radiopharmaceutical uptake in the 17 stress and resting segments, and the difference between the two (summed difference score-SDS). Uptake was considered improved if the resting study score dropped $\geq 1$ point relative to stress study. An SDS $\geq 4$ was considered positive (slight: SDS 4-8; moderate: SDS 9-12; severe SDS >12). Transient ischemic dilation on stress, a sign of subendocardial ischemia, was considered present if the ratio of stress-to-resting ventricular volume was $>1.2$.

Semiquantitative assessment of regional wall motion was performed by visual inspection of gated tomograms in cine-loop format. The left ventricular (LV) myocardium was divided into 17 segments and a 4-point scale was used for segmentary assessment of motion: 0 = normal; 1 = slightly hypokinetic; 2 = moderately hypokinetic; 3 = severely hypokinetic; and 4 = akinetic or dyskinetic. Observer-independent analysis was also conducted to assess ventricular function variables-regional motion, LVEF, and end-diastolic (abnormal if $>120 \mathrm{~mL}$ ) and end-systolic (abnormal if $>70 \mathrm{~mL}$ ) volumes-using Emory Cardiac Toolbox (ECTb) software. $\triangle$ LVEF was also considered (difference between poststress and resting LVEF).

For analysis of intraventricular synchrony, phase-derived indices were used, measured in resting and post-stress gated images by means of ECTb. The method has been well described.[7,8] The variables used to measure intraventricular synchrony were phase SD and histogram bandwidth. Cutoff values representing significant intraventricular dyssynchrony were $>43$ degrees for phase $\mathrm{SD}$ and $>135$ degrees for histogram bandwidth.[9]

Adverse events The patients included in the study were monitored by consultations and telephone calls (to patients or next of kin) at 1, 3, 6, 12, 24 and 36 months for the following adverse events: HF progression, potentially lethal ventricular arrhythmia, death from cardiac cause, death from noncardiac cause, myocardial revascularization, and acute coronary syndrome (STEMI or NSTEMI). Possible association between these events and ergometric, perfusion, myocardial, ventricular function, and intraventricular synchrony variables was assessed.

Data analysis Categorical variables were expressed as absolute and relative frequencies. To identify associations between these variables and dilated cardiomyopathy, the chi-square test or Fisher exact test was used. Continuous variables were summarized as the mean with SD. Kolmogorov-Smirnov and Shapiro-Wilks tests were used to assess whether variables were normally distributed. The next step depended on whether or not variables were normally distributed: in the case of normal distribution, a Student $t$ test was applied to the comparison groups for paired and unpaired observations, and in cases of non-normal distribution, the Mann-Whitney $U$ test and Wilcoxon signed-ranks test for quantitative variables were used. Chi square was used for qualitative variables.

The Kaplan-Meier method was used to calculate event-free survival rates, and log-rank was used for comparison of survival curves. A $p$ of $<0.05$ was considered significant.

Ethics The study was approved by the ICCCV scientific council. Written informed consent was obtained from all participants and patient anonymity was maintained during data analysis. 


\section{RESULTS}

Patient characteristics Of the 52 study patients, 41 (78.8\%) had ischemic left ventricular dysfunction; 34 (65.4\%) had a history of acute myocardial infarction, primarily anteriorly located $(18,34.6 \%)$. Although there were more coronary risk factors in IHD patients than in non-IHD, the difference was not statistically significant. The most frequent risk factors in both groups were smoking, hypertension and dyslipidemia.

Treadmill stress test During exercise, $61.5 \%(32 / 52)$ of all patients studied showed no symptoms. Only $15.4 \%$ (8/52) experienced chest pain. ST depression on stress occurred in $17.3 \%$ (9/52); 8 of these 9 patients were in the ischemic group. During maximal stress, $32.7 \%(17 / 52)$ had arrhythmia (primarily ventricular extrasystoles); 13 of the 17 were in the ischemic group. On stress, no significant differences between the two groups were found in symptoms, ST abnormalities or presence of arrhythmia.

Percentage of maximal heart rate reached during maximal stress was similar in both groups: 92.9 (SD 9.6) vs. 95.2 (SD 6.8 ), ischemic vs. nonischemic patients, $p=0.4$. Patients with IHD reached slightly fewer METs, without statistical significance, 5.05 (SD 1.19) vs. 5.97 (SD 1.97), $p=0.06$; likewise for exercise time, $6.9 \min (S D 1.6)$ vs. $7.8 \min (S D 2.4), p=0.13$.

Table 1: Myocardial perfusion and ventricular function in HF patients

\begin{tabular}{|l|r|r|r|} 
Variable & \multicolumn{1}{|c|}{$\begin{array}{c}\text { Ischemic } \\
(\mathbf{n}=\mathbf{4 1})\end{array}$} & $\begin{array}{c}\text { Nonischemic } \\
(\mathbf{n}=11)\end{array}$ & \multicolumn{1}{c|}{$\begin{array}{c}\text { p } \\
\text { Value }\end{array}$} \\
\hline & \multicolumn{2}{|c|}{ Mean (SD) } & \\
\hline SRS & $14.43(6.28)$ & $6.45(3.77)$ & $<0.001$ \\
\hline SSS & $16.82(6.37)$ & $7.54(5.8)$ & 0.001 \\
\hline SDS & $2.39(4.89)$ & $1.09(3.7)$ & 0.034 \\
\hline TID & $1.08(0.12)$ & $1.09(0.1)$ & 0.69 \\
\hline Resting LVEF $(\%)$ & $33.7(4.8)$ & $32(5.07)$ & 0.2 \\
\hline Stress LVEF $(\%)$ & $32.4(6.1)$ & $33.27(7.5)$ & 0.8 \\
\hline LLVEF $(\%)$ & $-1.29(5.8)$ & $1.27(4.31)$ & 0.06 \\
\hline Resting EDV $(\mathrm{mL})$ & $189(53)$ & $218(70)$ & 0.26 \\
\hline Stress EDV $(\mathrm{mL})$ & $190(53)$ & $226(77)$ & 0.14 \\
\hline Resting ESV $(\mathrm{mL})$ & $126(43)$ & $150(58)$ & 0.51 \\
\hline Stress ESV $(\mathrm{mL})$ & $129(44)$ & $154(65)$ & 0.07
\end{tabular}

$\triangle$ LVEF: post-stress LVEF - resting LVEF

EDV: end-diastolic volume

ESV: end-systolic volume

$\mathrm{HF}$ : heart failure

LVEF: left ventricular ejection fraction

SDS: summed difference score

SRS: summed rest score

SSS: summed stress score

TID: transient ischemic dilation

Gated SPECT myocardial perfusion scintigraphy Table 1 shows the behavior of myocardial perfusion and ventricular function variables obtained by gated SPECT, according to HF etiology. All summed perfusion scores were significantly higher in ischemic patients. Perfusion defects were severe in $53 \%$ of patients, moderate in $25 \%$, and slight in $22 \%$. Slight defects were more common in nonischemic patients $(64 \%$ vs. $10 \%$ in ischemic patients), while severe defects were more frequent in ischemic patients ( $66 \%$ vs. $9 \%$ in nonischemic patients).

In contrast, ventricular function variables (LVEF and volumes) were not significantly different, even though nonischemic patients had slightly higher ventricular volumes. $\triangle$ LVEF was slightly lower in ischemic patients.

Intraventricular synchrony Table 2 shows values for variables indicative of intraventricular synchrony. Neither phase SD nor histogram bandwidth showed significant differences at rest between the two study groups; however, they were significantly different with stress. Mean values for both variables decreased with stress among nonischemic patients. This was not the case among ischemic patients, who showed an increase, primarily in histogram bandwidth. The difference, however, was not statistically significant.

None of the patients in the study had normal phase SD or histogram bandwidth values. With cutoff values of 43 degrees and 135 degrees, respectively, ischemic patients exceeded the cutoff value more than nonischemic patients; only $14.6 \%(6 / 41)$ of ischemics versus $36.4 \%$ (4/11) of nonischemics had phase SD $<43$ degrees at rest $(p=0.37)$, compared to $9.8 \%(4 / 41)$ of ischemics vs. $36.4 \%(4 / 11)$ of nonischemics on stress $(p=$ $0.034)$. With regard to histogram bandwidth, $21.9 \%(9 / 41)$ of ischemics vs. $36.4 \%(4 / 11)$ of nonischemics had values $<135$ degrees at rest $(p=0.7)$, compared to $14.6 \%(6 / 41)$ of ischemics vs. $54.5 \%$ of nonischemics $(6 / 11)(p=0.004)$ with stress.

\begin{tabular}{|l|r|r|r|}
\hline $\begin{array}{l}\text { Table 2: Intraventricular synchrony variables in HF patients } \\
\text { Variable }\end{array}$ & $\begin{array}{c}\text { Ischemic } \\
(\mathbf{n}=\mathbf{4 1})\end{array}$ & $\begin{array}{c}\text { Nonischemic } \\
(\mathbf{n}=11)\end{array}$ & $\begin{array}{c}\text { p } \\
\text { Value }\end{array}$ \\
\hline Resting PSD & \multicolumn{2}{|c|}{ Mean (SD) } & 0.06 \\
\hline Stress PSD & $63.4(19.8)$ & $50.18(16.98)$ & 0.002 \\
\hline Resting HB & $197.45(15.93)$ & $47(11.69)$ & 0.41 \\
\hline Stress HB & $213.6(56.02)$ & $177.9(77.8)$ & 0.003 \\
\hline
\end{tabular}

HB: histogram bandwidth (degrees)

HF: heart failure

PSD: phase standard deviation (degrees)

Followup After three years, $30.8 \%(16 / 52)$ of patients were event free. At two and three years into the study, 92.3\% (48/52) were alive (Figure 1). Adverse events occurred more often in the ischemic than the nonischemic group ( $59.1 \%$ vs. $27.3 \%)$. Figure 2 shows the three-year survival curve with event-free survival greater among nonischemic patients $(57.1 \%$ vs. $27.3 \%)$. The only variable revealing a significant association with event occurrence was $<5$ METs on stress test $(p=0.03)$, while resting phase SD showed only a tendency toward an association $(p=0.05)$.

HF progression was the most frequent adverse event, occurring in $30.8 \%(16 / 52)$ of patients. Three deaths were recorded (two from cardiac causes), all in ischemic patients. During the study period, two patients developed ventricular tachycardia and four patients had acute coronary syndrome. Four myocardial revascularizations were performed: two surgically and two via percutaneous transluminal coronary angioplasty. When patients were contacted for final followup, two were awaiting coronary surgery and one was awaiting percutaneous transluminal coronary angioplasty.

Abnormal values for $\triangle L V E F$, resting and stress phase SD, resting and stress histogram bandwidth, and METs ( $<5$ METs during stress) were more frequent among patients who developed adverse events during followup (53\%, 95\%, 94\%, 89\%, $81 \%$ and $53 \%$, respectively), than among those who did not. 
Figure 1: Survival in HF patients 36 months after gated SPECT, Kaplan-Meier curve $(n=52)$
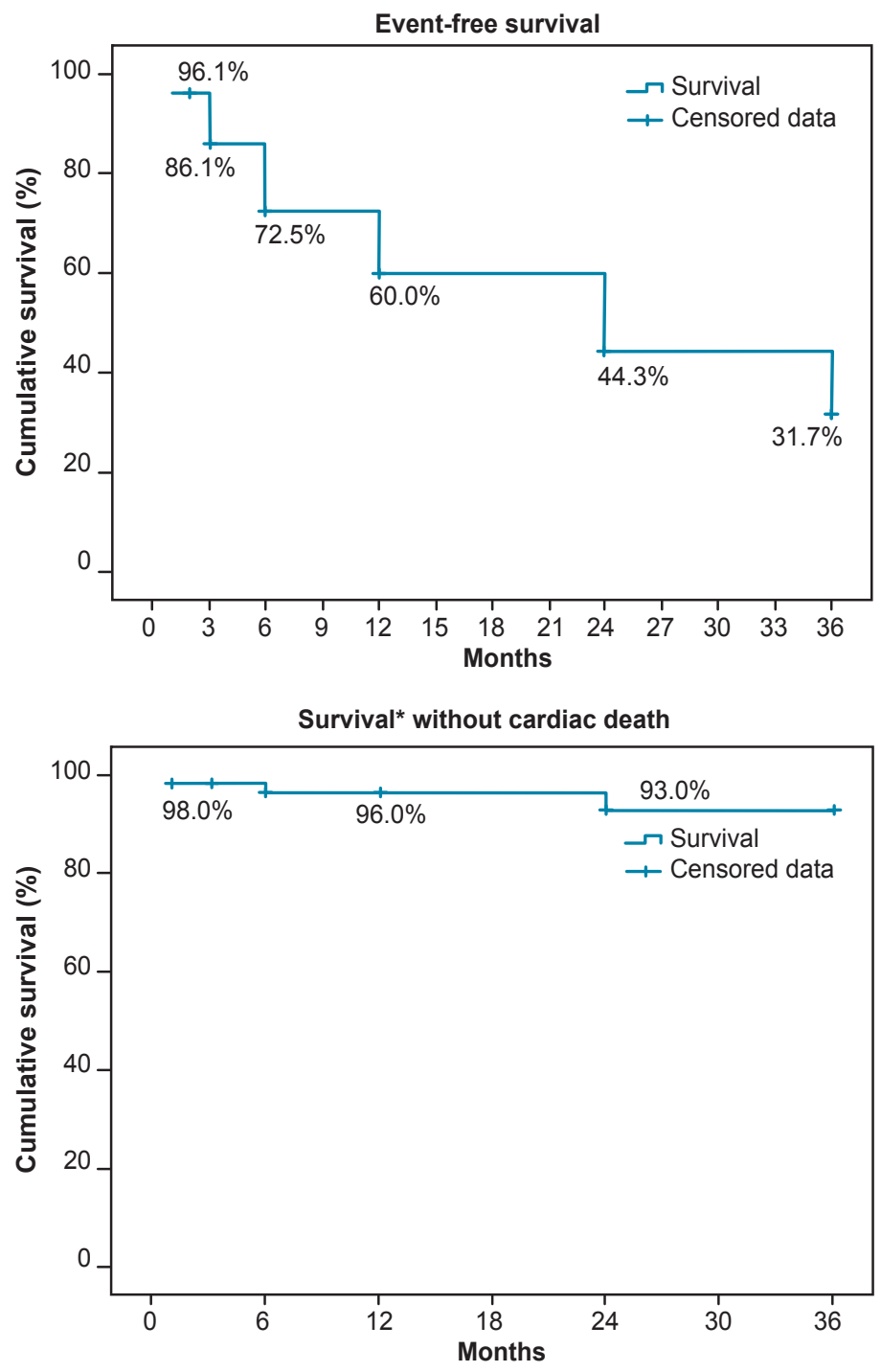

* Noncardiac death considered "survival" in this analysis

Figure 2: Event-free survival by diagnosis in HF patients 36 months after gated SPECT, Kaplan-Meier curve $(n=52)$

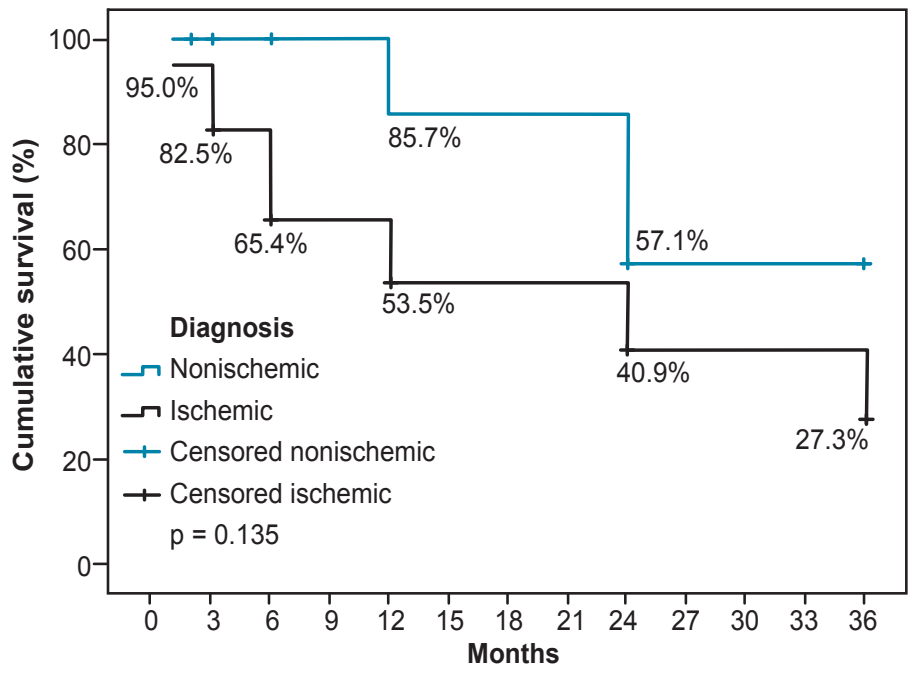

\section{DISCUSSION}

Although prognostic determinants for dilated cardiomyopathy patients have been studied, the value of stress-rest protocol gated SPECT has not yet been sufficiently established (including evaluation of perfusion, function and intraventricular synchrony) for risk stratification in these patients.

While it is known that resting LVEF is one of the most important variables for estimating HF patient prognosis, it is also true that other factors should be taken into account as well. This study found that IHD patients had more severe perfusion defects and a slightly greater drop in LVEF than patients without IHD. No significant changes in variables that measure intraventricular synchrony were found between resting and stress within each patient group (with or without IHD diagnosis). IHD patients, however, became more asynchronous on stress than those without IHD, compared to resting values. They also showed a higher degree of asynchrony on stress. The two variables that showed a possible association with development of adverse events during followup were low cardiovascular functional capacity with stress ( $<5 \mathrm{METs}$ ) and resting phase SD.

In a study of 167 patients with ischemic dilated cardiomyopathy, Candell-Riera found that an independent predictor of cardiac death in the resting SPECT study was presence of zones of viable tissue in the infarcted area; this was also a predictor on stress SPECT, along with ischemia in noninfarcted areas and exercise duration $\leq 5$ minutes.[6] In our case, the fact that these variables did not show a significant association with adverse event occurrence on followup could be due to the small sample size.

In addition, the lower functional capacity on stress associated with events in our study was also related to shorter exercise time. Intraventricular synchrony variables were not analyzed in the CandellRiera study. Our group conducted a previous study using resting gated SPECT perfusion scintigraphy on dilated cardiomyopathy patients, which also assessed perfusion, function and synchrony, but did not consider the effect of exercise on these variables.[14] It was found that presence of ischemia, SRS and phase SD were the variables that showed greatest association with adverse event occurrence during followup, which supports our current results concerning abnormal resting-phase SD.

Gated SPECT myocardial perfusion scintigraphy Myocardial perfusion scintigraphy may contribute to differential diagnosis of ventricular dysfunction based on etiology, given that ischemic perfusion defects in IHD patients are generally diffuse, extensive and severe and follow the distribution of territories corresponding to coronary arteries; and that they coexist with segmental-wall motion abnormalities, usually coinciding with territories having perfusion deficits. Cases of nonischemic etiology, however, show homogeneous radiopharmaceutical uptake or slight to moderate perfusion defects that do not follow a coronary distribution, along with diffuse motion abnormalities.[16]

Such observation are consistent with our results, where all summed perfusion scores were significantly higher in IHD patients. Additionally, slight defects were significantly more frequent in nonischemic patients, while severe defects were more frequent in the ischemic group. Regarding ventricular function, although no significant differences were found, it is noteworthy that $\triangle L V E F$ was slightly lower (more negative) in ischemic patients, related to a 
greater tendency to decreasing LVEF with stress as a manifestation of myocardial stunning and, therefore, of ischemia.

Intraventricular synchrony It has been shown that variables that measure intraventricular dyssynchrony (phase SD and histogram bandwidth) do not vary between rest and stress in the same patient, even when ischemia is present.[12] In general, this matches our results where no significant rest-stress differences were found. However, our IHD patients tended to be more asynchronous on stress. For this reason, it is important to remember that images labeled with technetium-99m compounds are typically taken one hour following stress. Perfusion reflects the moment the radiopharmaceutical is injected because these compounds do not redistribute; however, function and synchrony are measured in real time and therefore reflect one-hour post stress rather than maximal stress. Thus, if images were obtained closer to maximal stress (15 to 20 minutes), a greater difference could be found, representing ischemia-induced dyssynchrony. This has already been raised by some authors, both through use of thallium-201,[17] with images taken 5-10 minutes following stress, and through earlier imaging labeled with technetium-99m compounds.[18]

AlJaroudi found no correlation between defect size (representing extent of ischemia) and change in phase SD and histogram bandwidth between rest and stress.[12] However, this study did find significant differences in phase SD and histogram bandwidth on stress in both groups: ischemic patients were more dyssynchronous than nonischemics, which is consistent with other authors' findings regarding myocardial ischemia as a producer of dyssynchrony.[17-20]

Ischemic and infarcted segments exhibit sizeable degrees of segmental wall motion abnormalities, which result in abnormal contraction and dyssynchrony.[18,21] In this regard, Chen showed that stress-induced ischemia causes dyssynchronous contraction in the ischemic area, while normal myocardium has more synchronous contraction during stress.[17] Hida, in a study of 278 IHD patients, found that although an SSS of $\geq 9$ showed greater sensitivity in detection of multivessel disease, a stress-induced increase in phase SD of $\geq 4.4$ degrees and a histogram bandwidth of $\geq 14$ degrees had specificities of $84 \%$ and $91 \%$, respectively, and accuracy of $80 \%$ (the highest of all analyzed perfusion and function variables).[18]

Another study that supports our results was conducted by Vidigal of 143 patients with LVEF $\leq 50 \%$, in which patients with mechanical dyssynchrony had a greater prevalence of abnormal perfusion, with extensive defects, increased end-diastolic volume and worse LVEF.[20]
Predictors of events during followup Ischemic etiology of HF related to presence of dyssynchrony is an important factor to consider as a predictor of adverse events in HF patients.[22] Several authors[10,23,24] have shown that SRS is an independent predictor of mechanical dyssynchrony, which is understandable, considering that ischemic and infarcted segments with segmental wall motion abnormalities lead to a dyssynchronous pattern.[25] This coincided with our results in a previous study of dilated cardiomyopathy patients who underwent rest-only scintigraphy,[15] where the greatest ORs for prediction of events were 1.91 (phase $\mathrm{SD}$ ), 1.66 (ischemic etiology) and 1.55 (SRS); $89 \%$ of adverse events on followup occurred in the ischemic group.

This study introduces assessment of physical exertion as a stressor, which is not always possible in these patients because of their marked functional impairment. A limitation in our study was the small sample size, especially in the nonischemic group, influenced by the relatively small number of ventricular dysfunction patients referred for stress testing.

It is important to note, however, that although statistical significance was not found (probably due to sample size), there is clinical significance in the fact that abnormal values for $\triangle L V E F$, resting and stress phase $S D$, resting and stress histogram bandwidth and METs ( $<5$ METs during stress) were more frequent among patients who experienced adverse events during followup.

AlJaroudi, using stress-rest protocol positron emission tomography in 486 patients with LVEF of 26 (SD 6\%), found that dyssynchrony was an independent predictor of mortality, and in turn that perfusion defect size, reduced resting LVEF and absence of contractile reserve were predictors of worse dyssynchrony at peak stress.[26] This coincided with our findings in ischemic patients.

Even though there was no significant difference in occurrence of adverse events between IHD and nonischemic patients in our study, which could be influenced by small sample size (the abovementioned limitation), we believe that the finding of a much greater occurrence of adverse events during followup in IHD patients compared with non-IHD patients does have clinical value.

\section{CONCLUSIONS}

Information on myocardial perfusion, functional capacity and intraventricular synchrony obtained through rest-stress gated SPECT may help identify patients with symptomatic heart failure who have a greater probability of developing adverse cardiac events, enabling better allocation of medical resources and management of higher-risk cases. $-1 /$ -

\section{REFERENCES}

1. Giubbini R, Milan E, Bertagna F, Mut F, Metra $M$, Rodella C, et al. Nuclear cardiology and heart failure. Eur J Nucl Med Mol Imaging. 2009 Dec;36(12):2068-80.

2. Mann DI. Management of heart failure patients with reduced ejection fraction. In: Bonow RO, Mann DL, Zipes DP, Libby P, editors. Braunwald's Heart Disease. A textbook of cardiovascular medicine. 9th ed. Philadelphia: Elsevier Saunders; 2011. p. 543-77.

3. Bleumink GS, Knetsch AM, Sturkenboom MC, Straus SM, Hofman A, Deckers JW, et al. Quantifying the heart failure epidemic: prevalence, incidence rate, lifetime risk and prognosis of heart failure. Eur Heart J. 2004 Sep;25(18):1614-9.

4. Armas Rojas NB, Dueñas Herrera A, de la Noval García R, Castillo Guzmán A, Suárez Medina R, Varona Pérez $\mathrm{P}$, et al. Enfermedades del corazón y sus características epidemiológicas en la población cubana de 15 años y más. Rev Cub Inv Biomed [Internet]. 2009 [cited 2013 Jul 26];28(4). Available from: http://scielo.sld.cu/scielo.php?pid=S0864-0300 2009000400004\&script=sci arttext. Spanish

5. Atchley $A E$, Iskandrian $A E$, Bensimhon $D$, Ellis SJ, Kitzman DW, Shaw LK, et al. Relationship of technetium-99m tetrofosmin-gated rest single-photon emission computed tomography myocardial perfusion imaging to death and hospitalization in heart failure patients: results from the nuclear ancillary study of the HF-ACTION trial. Am Heart J. 2011 Jun;161(6):1038-45.

6. Candell-Riera J, Romero-Farina G, AguadéBruix S, Castell-Conesa J, de León G, GarcíaDorado D. Prognostic value of myocardial perfusion-gated SPECT in patients with ischemic cardiomyopathy. J Nucl Cardiol. 2009 MarApr;16(2):212-21.

7. Chen J, Garcia EV, Folks RD, Cooke CD, Faber TL, Tauxe EL, et al. Onset of left ventricular mechani- 
cal contraction as determined by phase analysis of ECG-gated myocardial perfusion SPECT imaging: Development of a diagnostic tool for assessment of cardiac mechanical dyssynchrony. J Nucl Cardiol. 2005 Nov-Dec;12(6):687-95.

8. Chen J, Garcia EV, Bax JJ, Iskandrian AE, Borges-Neto S, Soman P. SPECT myocardial perfusion imaging for the assessment of left ventricular mechanical dyssynchrony. J Nucl Cardiol. 2011 Aug;18(4):685-94.

9. Henneman MM Chen J Dibbets-Schneider P, Stokkel MP, Bleeker GB, Ypenburg C, et al. Can LV dyssynchrony as assessed with phase analysis on gated myocardial perfusion SPECT predict response to CRT? J Nucl Med. 2007 Jul;48(7):1104-11.

10. Samad Z, Atchley AE, Trimble MA, Sun JL, Shaw LK, Pagnanelli R, et al. Prevalence and predictors of mechanical dyssynchrony as defined by phase analysis in patients with left ventricular dysfunction undergoing gated SPECT myocardial perfusion imaging. J Nucl Cardiol. 2011 Feb;18(1):24-30.

11. Trimble MA, Borges-Neto S, Honeycutt EF, Shaw LK, Pagnanelli R, Chen J, et al. Evaluation of mechanical dyssynchrony and myocardial perfusion using phase analysis of gated SPECT imaging in patients with left ventricular dysfunction. J Nucl Cardiol. 2008 Sep-Oct;15(5):663-70.

12. Aljaroudi W, Koneru J, Heo J, Iskandrian AE. Impact of ischemia on left ventricular dyssynchrony by phase analysis of gated single photon emission computed tomography myocardial perfusion imaging. J Nucl Cardiol. 2011 Feb;18(1):36-42.

13. AlJaroudi W, Jaber WA, Cerqueira MD. Effect of tracer dose on left ventricular mechanical dyssynchrony indices by phase analysis of gated single photon emission computed tomography myocardial perfusion imaging. J Nucl Cardiol. 2012 Feb;19(1):63-72.

14. Peix A, Karell J, Rodríguez L, Cabrera LO, Padrón K, Carrillo R, et al. Gated SPECT myocardial perfusion imaging, intraventricular synchronism, and cardiac events in heart failure. Clin Nucl Med. 2014 Jun;39(6):498-504.

15. Cerqueira MD, Weissman NJ, Dilsizian V, Jacobs AK, Kaul S, Laskey WK, et al. Standardized myocardial segmentation and nomenclature for tomographic imaging of the heart. A statement for healthcare professionals from the Cardiac Imaging Committee of the Council of Clinical Cardiology of the American Heart Association. Circulation. 2002 Jan 29;105(4):539-42.

16. Ananthasubramaniam K, Dhar R, Cavalcante JL. Role of multimodality imaging in ischemic and non-ischemic cardiomyopathy. Heart Fail Rev. 2011 Jul:16(4):351-67.

17. Chen CC, Shen TY, Chang MC, Hung GU, Chen WC, Kao $\mathrm{CH}$, et al. Stress-induced myocardial ischemia is associated with early poststress left ventricular mechanical dyssynchrony as assessed by phase analysis of 201TI gated SPECT myocardial perfusion imaging. Eur J Nucl Med Mol Imaging. 2012 Dec;39(12):1904-9.

18. Hida S, Chikamori T, Tanaka H, Igarashi $Y$, Shiba $\mathrm{C}$, Usui $\mathrm{Y}$, et al. Diagnostic value of left ventricular dyssynchrony after exercise and at rest in the detection of multivessel coronary artery disease on single-photon emission computed tomography. Circ J. 2012 May;76(8):1942-52

19. Murrow J, Esteves F, Galt J, Chen J, Garcia E, Lin J, et al. Characterization of mechanical dyssynchrony measured by gated single photon emission computed tomography phase analysis after acute ST-elevation myocardial infarction. J Nucl Cardiol. 2011 Oct;18(5):912-9.

20. Vidigal Ferreira MJ, Silva R, Cabanelas N, Cunha MJ, Ramos D, Albuquerque A, et al. Left ventricular dyssynchrony in patients with impaired left ventricular function undergoing gated SPECT myocardial perfusion imaging. Rev Port Cardiol. 2013 May;32(5):387-94

21. Ramakrishna G, Miller TD, Hodge DO, O'Connor MK, Gibbons RJ. Differences in left ventricular ejection fraction and volumes measured at rest and poststress by gated sestamibi SPECT. J Nucl Cardiol. 2006 Sep;13(5):668-74.

22. Fang $\mathrm{W}$, Zhang J, He ZX. Myocardial ischemia in patients with dilated cardiomyopathy. Nucl Med Communicat. 2010 Nov;31(11):981-4.

23. Bader H, Garrigue S, Lafitte S, Reuter S, Jaïs $P$, Haïssaguerre $\mathrm{M}$, et al. Intra-left ventricular electromechanical asynchrony. A new independent predictor of severe cardiac events in heart failure patients. J Am Coll Cardiol. 2004 Jan 21:43(2):248-56.

24. Cho GY, Song JK, Park WJ, Han SW, Choi SH, Doo YC, et al. Mechanical dyssynchrony assessed by tissue Doppler imaging is a powerful predictor of mortality in congestive heart failure with normal QRS duration. J Am Coll Cardiol. 2005 Dec 20;46(12):2237-43.

25. Forrester JS, Wyatt HL, Da Luz PL, Tyberg JV, Diamond GA, Swan HJ. Functional significance of regional ischemic contraction abnormalities. Circulation. $1976 \mathrm{Jul} ; 54(1): 64-70$.

26. AlJaroudi W, Alraies MC, Menon V, Brunken RC, Cerqueira MD, Jaber WA. Predictors and incremental prognostic value of left ventricular mechanical dyssynchrony response during stressgated positron emission tomography in patients with ischemic cardiomyopathy. J Nucl Cardiol. 2012 Oct:19(5):958-69.

\section{THE AUTHORS}

Amalia Peix (Corresponding author: peix@ infomed.sld.cu), cardiologist with doctorate in medical sciences and advanced doctorate in science. Full professor and senior researcher, Cardiology and Cardiovascular Surgery Institute (ICCCV), Havana, Cuba.

Yanisbel Macides, dual specialist in family medicine and cardiology, ICCCV, Havana, Cuba.

Lydia Rodríguez, adjunct researcher with degrees in computer science and mathematics, ICCCV, Havana, Cuba.

Lázaro O. Cabrera, dual specialist in family medicine and cardiology. Assistant professor and adjunct researcher, ICCCV, Havana, Cuba.

Kenia Padrón, dual specialist in family medicine and cardiology Assistant professor and adjunct researcher, ICCCV, Havana, Cuba.

Flor Heres, immunologist with master's degree in atherosclerosis. Assistant professor and associate researcher, ICCCV, Havana, Cuba.

Dania Consuegra, radiation protection supervisor with degree in nuclear physics, ICCCV, Havana, Cuba.

Yoel Fernández, nuclear medicine technologist, ICCCV, Havana, Cuba.

Erick Mena, nuclear medicine technologist, ICCCV, Havana, Cuba.

Submitted: August 14, 2013

Approved for publication: March 26, 2015 Disclosures: None 